\title{
Early manifestations of manual specialisation in infants: A longitudinal study from 20 to 30 weeks
}

\author{
F. Morange-Majoux , C. Lemoine \& G. Dellatolas
}

To cite this article: F. Morange-Majoux , C. Lemoine \& G. Dellatolas (2013) Early manifestations of manual specialisation in infants: A longitudinal study from 20 to 30 weeks, Laterality:

Asymmetries of Body, Brain and Cognition, 18:2, 231-250, DOI: 10.1080/1357650X.2012.660163

To link to this article: https://doi.org/10.1080/1357650X.2012.660163

\section{Published online: 01 Jun 2012.}

Submit your article to this journal ๘

Џ Article views: 106

Q View related articles $\triangle$

Citing articles: 8 View citing articles ๘ 


\title{
Early manifestations of manual specialisation in infants: A longitudinal study from 20 to 30 weeks
}

\author{
F. Morange-Majoux, C. Lemoine, and G. Dellatolas \\ Laboratoire de Psychologie et Neuropsychologie Cognitive \\ (FRE 3292), Université Paris Descartes - CNRS, \\ Boulogne-Billancourt, France
}

This longitudinal study examined lateral differences between latency time of the two hands during the development of prehension in 12 infants from 20 to 30 weeks. Latency time (LT) is defined as the delay between the visual localisation of a reachable target and the beginning of the movement and could be considered as a phase in the preparation of action. If LT varies with the hand used and the type of movement, this would suggest differences in information processing (nature and/or quantity). Results show that the latency time is shorter for the left hand approach movements and shorter for the right hand grasping movements. These findings are in favour of a manual specialisation - clearly present from 20 weeks - and are discussed in a possible hemispheric specialisation perspective.

Keywords: Handedness; Latency time; Manual specialisation; Development of prehension.

A grasping movement requires two phases: a rapid phase devoted to projecting the arm towards the object, and a slow phase devoted to pointing accurately at the object and adjusting the fingers on it for grasping (Annett, 2004; Flowers, 1975; Jeannerod, 1981; Jeannerod, Paulignan, \& Weiss, 1998; Todor \& Doane, 1978; Von Hofsten, 1991). The rapid phase requires coding of spatial parameters such as direction and amplitude, and temporal parameters such as velocity; the slow phase is devoted to coding according to the object's size and shape (Gentilucci et al., 1991).

To be a success (catch the object) these movements involve a preparatory phase from the perceptual apprehension of the target object, which

Address correspondence to: Françoise Morange-Majoux, Laboratoire de Psychologie et Neuropsychologie Cognitive (FRE 3292), Université Paris Descartes - CNRS, 71 avenue Edouard Vaillant, 92774 Boulogne-Billancourt Cedex, France. E-mail: francoise-morange-majoux@, parisdescartes.fr 
corresponds to the latency period of the hand movement (Carson, Chua, Goodman, Byblow, \& Elliott, 1995). The duration of this preparatory phase, called reaction time (RT), is defined as a latency preceding the goal-directed movement when the participant is asked to react as rapidly as possible. It is supposed to allow the person to gather and process information, particularly visual information about the position of object and the object itself, and also kinaesthetic and proprioceptive information for the position of the arm and fingers of the hand before the hand begins to move. In adults it has been shown that RT is affected by properties of the target object and the precision requirements of the task (Klatzky, Fikes, \& Pellegrino, 1995; Pellegrino, Klatzky, \& McCloskey, 1989). Henry and Rogers (1960) were the first to document that the time taken to initiate a movement, as RT, is longer for more complex movements (i.e., one finger lift vs sequence of several movements of the fingers). When the stimulus is held constant, increases in RT are viewed as reflecting the additional time required to program more complex movements (Sidaway, Yook, \& Russell, 1999). In infants latency is also observed before the beginning of the movement; however, as infants cannot be asked to react as rapidly as possible, we will use the term latency time (LT) instead of reaction time. The infant literature on this topic focuses more on changes in accuracy of reaching/grasping movements than on the LT, i.e., the preparatory phase, of pre-reaching and reaching movements, with some exceptions. Brown, Sepher, Ettlinger, \& Skreczek (1986) and Bard, Hay, and Fleury (1990) show that LT for reaching clearly decreases between 2 and 10 years. In 6- to 8-month-old infants, Fagard (1997) compared successful and unsuccessful grasps and reported that the longer the LT, the more successful the grasp. This suggests that LT is a preparatory phase for grasp which becomes more and more automatic during development and requires a shorter LT. Also, visual perception investigations have reported that saccade latencies decrease with experience in 4-month-old infants suggesting progressive automatisation (Johnson, Posner, \& Rothbart, 1994). We postulate that LT may have the same meaning in children as in adults; that is, LT reflects the time necessary for information processing preparing the movement. Thus it seems important to investigate the LT further, according to the deep functional changes in the development of prehension, especially during the weeks preceding and following the onset of prehension.

The period from 4 to 7 months is particularly rich in developmental changes (Berthier, Clifton, McCall, \& Robin, 1999; McDonnell, 1975; Morange \& Bloch, 1996; Rönnqvist \& Domellof, 2006; von Hofsten, 1991), and reaching is considered to be an essential skill acquired during the early development of goal-directed actions (Fallang, Saugstad, Grogaard, \& Hadders-Algra, 2003). Development of infant arm movements is gradually settled. Actions appear in the following order: first, approach movements to localise the object (arm and hand movements directed towards 
an object without any contact), are performed by infants from 13 to 20 weeks (Bruner, 1969; DiFranco, Muir, \& Dodwell, 1978; McDonnell, 1975; von Hofsten, 1982, 1991); then reaching (arm and hand movements that end in contact with the object but do not grasp it); followed by grasp movements (successful prehension movements, with fingers adjusted to the object and the hand surrounding it). The latter two appear successively, around 20-25 weeks respectively, and allow manipulation of objects (Thelen, Corbetta, \& Spencer, 1996).

These stages can be interpreted within Jeannerod's conceptual framework of adult reaching movements: approach movements are based only on visual information about the object's location, which can be perceived and encoded quickly. Thus we can hypothesise that an approach movement will be initiated from a rapid visual apprehension of the object. On the other hand, reaching and grasp movements, which appear later, require a more complex perceptual apprehension; the person not only has to determine a general direction for his/her hand movement, but he/she must also consider the precise distance of the object and some qualities as size, volume, shape, and orientation in order to determine and anticipate the best angle to initiate the movement. We can presume that such actions would require a longer initial visual inspection before the hand begins to move (Fish \& Goodale, 1979; Henry \& Rogers, 1960; Pratt \& Abrams, 1996). Berthier (1996) presented a mathematical model in which it is assumed that infant reaches are a sequence of corrective submovements. LT analysis during this period could reflect changes in information processing that are a function of the type of movement.

Between 4 and 7 months infants exhibit hand movements that already manifest laterality, as identified by relative differences in frequency of use (Michel \& Harkins, 1986). Most studies on laterality in infants have focused on the timing of first appearance of right-hand preference, at around 6 months (for a review see Michel, 2002) and more rarely on manual specialisation. However, as Michel pointed out (1998) the clearly observable hand-use preference involves or reveals differences in functioning between the cerebral hemispheres. In this perspective we have observed some specific lateral differences according to the type of movement used at these ages (Morange \& Bloch, 1996; Morange-Majoux, Pezé, \& Bloch, 2000): approach movements are mostly performed by the left hand; reaching and grasp movements are mostly performed by the right hand. We have suggested that the left hand is more involved in spatial abilities and the right hand in accuracy abilities (Morange-Majoux et al., 2000).

In adults many studies concerning manual specialisation have shown a specialisation at the level of (i) manual execution: a left hand advantage in visuo-spatial tasks and a better accuracy with the right hand (Elliott \& Chua, 1996; Fisk \& Goodale, 1985); (ii) LT: the left hand's RT is shorter than 
the right hand's (Boulinguez, Nougier \& Velay, 1991; Carson, Chua, Elliott, \& Goodman, 1990; Carson et al., 1995; Elliott et al., 1993; Haaland \& Harrington, 1989; Velay, Daffaure, Raphael, \& Benoit-Dubrocard, 2001). However, most of these studies in adults used computer screens or switches and did not necessary involve grasping. Thus the shorter latency time for the left hand has been explained by the right hemisphere's greater spatial planning ability and/or the allocation of attention in space (Carson, 1989; Hodges, Lyons, Cockell, Reed, \& Elliott, 1997). Still, hitting a screen or switch simply requires a ballistic movement; the difference between the two hands could then be interpreted as the left hemisphere operating with a ballistic movement and the right hemisphere with sensory-guided movements.

In a study in infants aged 4 months Morange-Majoux and Dellatolas (2010) observed left and right hand movements towards a midline object in a constrained condition where the hands were maintained closed (the two hands were introduced in small rubber socks, maintaining the hands closed but keeping free all other upper-limb movements) and in a free condition. Two types of movements were coded: approach movements (directed towards the object but without a hand contact with the object) and reaching movements (directed towards the object with a hand-object contact). A left hand dominance for approach movements and a right hand dominance for reaching movements were observed in the free condition. In the constrained condition reaching movements were strongly reduced. Concerning approach movements, results showed clear manual specialisation: approach movements were more frequent with the left hand even if the hand is maintained closed; by contrast, right approach movements were strongly reduced when the hand was maintained closed. This was interpreted as evidence that right (but not left) arm movements are pre-prehension movements at an age where prehension is not yet possible.

The aim of the present longitudinal study is to extend and complete these results, more specifically: (i) to investigate the passage from approach to reaching and grasping of the left and right hands in infants aged from 20 weeks to 30 weeks (prehension acquisition period); (ii) to investigate LT of each type movement (approach, reaching, grasping) of the left and right arms. For instance, if approach movements of the right arm are in fact prereaching/grasping movements, their LT should be longer than that of approach movements of the left arm. Conversely, LT for grasping movements might be shorter for the right arm than the left arm. To accomplish these specific aims, a longitudinal study from 20 to 30 weeks was carried out to examine LT as a function of age and type of hand movement. Objects were placed laterally, not at the midline, to observe all types of movements by both hands. 


\section{METHOD}

\section{Participants}

We conducted a longitudinal study with 12 babies ( 6 girls and 6 boys) from 20 to 30 weeks. The infants were examined every two weeks $(20,22,24,26$, 28, and 30 weeks). All infants were of European origin, born full-term, with no known or suspected abnormalities, and were from middle-class families. They were recruited in day-care centres in Paris, from a list of local families who expressed interest in being part of infant development studies. Infants were tested at the day-care centre in a quiet room after parental consent had been granted. The experiment was conducted in accordance with the ethical standards specified in the 1964 Declaration of Helsinki. The parents were all right-handed according to the Edinburgh inventory (Oldfield, 1971). Thus it was expected that no more than 2 (max. 3) of these 12 children would become left-handers. Possible future left-handedness cannot be affirmed with confidence at this age (Michel, Tyler, Ferre, \& Sheu, 2006).

\section{Procedure}

Infants were observed in a quiet room, while seated and attached in a comfortable baby chair at maximum vertical position $\left(90^{\circ}\right)$, with body straight and arms free to move, in front of a table at the height of the torso. The body was maintained against the back of the baby chair with belts in order to minimise the role of postural instability in arm movements (Rochat, 1992). This position also limited head movements without the necessity of additional head support. The object presented was a red cube of $2.6 \mathrm{~cm}$ each side, placed on the table within reach $(12 \mathrm{~cm})$, to the right or to the left of the child (at $45^{\circ}$ to his/her antero-posterior axis), in order to induce ipsilateral movements (Fagard \& Lockman, 2005; Morange \& Bloch, 1996). The distance between the infant and the object was individually adjusted to enable the baby to reach the object by extending the arm. At the start of each trial the first experimenter, standing behind the baby, placed the baby's hands near his/her body (standardised position); a large piece of cardboard $(40 \times 60 \mathrm{~cm})$ was positioned in front of the baby and the object was placed behind the cardboard, on the table, in the left or right position (the cardboard was large enough to hide both possible places of the object), by a second experimenter. At this point the object and its position were hidden by the cardboard. Then the first experimenter released the baby's hands and the second experimenter removed the cardboard, uncovering the object (this procedure lasted a few seconds). The trial began when the experimenter began lifting the cardboard and ended when the infant had either made an approach, reaching, or grasping movement (see analysis section below for definition of each type of movement), or did not look at the object for more 
than 20 seconds. For each participant there were 10 trials, with the object equally distributed alternately in the right and left sides in a counterbalanced order among the participants. At the end of each trial an experimenter distracted the baby with toys. After this little pause, a new trial began.

Criteria of inclusion for movements were unimanual and ipsilateral movements directed towards the object under visual control. Some trials (23 in all) were discarded after a few sessions because movements were either bimanual or contralateral to the object position. However, a maximum of one trial per baby and per test session was discarded.

\section{Data collection}

All movements were captured by two cameras, placed perpendicular to each other, one at 1 metre in front of the infant, the other at 1 metre above the head of the infant. The cameras were synchronised at the start of each session by recording an index-thumb closing movement in front of the cameras that was fast enough for a single image to be identified on each camera. Time-position data of the infants' upper arm movements were sampled at $25 \mathrm{~Hz}$ (25 images per second). Duration of gaze, identification of hand used, and type of movement were coded frame by frame.

\section{Analysis}

Only unimanual, ipsilateral movements performed when the infants were looking at the object were scored. Movements not in the direction of the object, like excitation movements, were not considered. The analyses of parameters are based on 697 movements in total (12 babies $\times 6$ ages $\times 10$ trials minus 23 trials) and all were analysed frame by frame ( 350 right arm movements, 347 left arm movements). In accordance with Morange and Bloch's previous classification (1996), hand movements were scored and classified into three categories: (1) approach movement: movement with no object contact; (2) reaching movement: movement with object contact; and (3) grasp movement: movement with grasp of object. A movement was defined as an arm movement extended forward $10 \mathrm{~cm}$ or more, directed towards the object; the start of the movement was the first frame where the arm moved. These movements were defined by their end. As regards the approach movement, the end of the movement was defined by the hand stopping still near the object at $5 \mathrm{~cm}$ or less. The hand either stops on the table for more than three frames or is withdrawn. This movement was very fast. For reaching, the end of the movement was defined as the first frame where the object was touched by the hand, without attempt to grasp the object. Grasp movements were either attempts to grasp the object based on the shape of the hand or successful grasps. The three categories were 
exclusive so a single movement could not be classified in two categories. Ambiguous movements (about 1\%) were excluded (rare reaching movements could have breaks of three to four frames and could be interpreted as approach movements).

LT was defined as the visual latency (in seconds) between the baby's first look at the object after the cardboard was removed, and the start of the hand movement. The baby's first look was determined from the frames of the camera facing the infant. LT was examined for each age and each movement. The manual data were scored from the frontal camera and the overhead camera frame by frame. A second coder also analysed $50 \%$ of the movements (350 movements) chosen at random to assess the reliability of coding for LT and type of movement. The inter-rater reliability of the two coders was $96 \%$ for LT coding and $97 \%$ for movement coding. Contentious movements and/or LTs were re-examined by both experimenters and re-coded together until a $100 \%$ inter-rater agreement was eventually reached.

\section{Statistical analyses}

Repeated-measures analyses were performed using the General Linear Model (GLM) procedure of the SAS software, which gives multivariate test statistics for within-participant effects. In a first step dependent variables were proportions of approach, reaching, and grasping movements. Separate analyses were performed for each type of movement. Within-participant factors were age (six levels: 20, 22, 24, 26, 28, and 30 weeks) and hand (two levels: right, left). In a second step the dependent variable was LT (mean LT of the five trials per hand) and within-participant factors age (six levels) and hand (two levels). Indeed, a preliminary analysis with age, hand, and trial (five trials per hand) as within-participant factors did not show any significant effect of trial. In a third step an ANOVA at 20 weeks explored the effects of hand (two levels: right, left) and type of movement (two levels: approach, reaching) on LT. This allowed us to compare LT of approach and reaching, as no grip movements were present at 20 weeks. Individual data for LT of approach movements at 20 weeks for the left and right arm are also presented. Finally, in a fourth step LTs were calculated by type of movement (i.e., LT for approach, reaching, and grasping movements), and GLM analyses with movement-specific LT as dependent variable were performed. These analyses were limited by the absence of some types of movements at the beginning or the end of the follow-up period (e.g., absence of grasping at 20 weeks; absence of right arm approach after 22 weeks). LT of reaching movements were analysed according to hand (two levels) and age (three levels: 20, 22, 24 weeks), and LT of grasping movements were analysed according to hand (two levels) and age (four levels: 24, 26, 28, 30 weeks). 


\section{RESULTS}

\section{Type of movement in relation to age and hand}

Figure $1(a, b, c)$ shows the proportion of each type of movement by hand and age. For instance, at 20 weeks there are $45 \%$ approach and $55 \%$ reaching movements with the left hand, and $25 \%$ and $75 \%$ with the right hand. Repeated-measures ANOVAs on the proportion of each type of movement showed a significant effect of age in all cases-approach: $F(5,7)=48.2$, $p<.001$; reaching: $F(5,7)=554.6, p<.001 ;$ grasping: $F(5,7)=285.6$, $p<.001$ - and a significant hand effect for approach movements only: more approach movements with the left arm than with the right hand, Wilk's Lambda $=0.0283, F(1,11)=9.94, p=.009$. Age by hand interaction did not reach significance. Detailed analysis of the age effect for each movement was performed: (1) for approach movements, a significant decrease from 20 to 22 weeks was observed, $F(1,11)=70.6, p<.001$, as well as a linear trend towards decrease from 22 to 30 weeks, $F(4,8)=9.5, p=.004$, without other significant difference between two successive periods; (2) for reaching movements, comparison between two successive periods showed significant decrease from 24 to 26 weeks, $F(1,11)=13.0, p=.004$, and from 26 to 28 weeks, $F(1,11)=14.0, p=.003$; (3) for grasping movements, comparison between two successive periods showed significant increase from 20 to 22 weeks, $F(1,11)=45.9, p<.001$, from 22 to 24 weeks, $F(1,11)=9.3, p=.01$, from 24 to 26 weeks, $F(1,11)=18.2, p=.001$, and from 26 to 28 weeks, $F(1,11)=12.0, p=.005$. In sum, right hand approach movements are rare and disappear completely after 22 weeks. Reaching movements predominate at 20,22 , and 24 weeks, but become rare at 28 and 30 weeks. Grasping movements are completely absent at 20 weeks, and represent more than $90 \%$ of all movements of the right and left arms/hands at 30 weeks

\section{$\mathrm{LT}$ in relation to age and hand}

A repeated-measures ANOVA with age and hand as within-participant factors showed a significant effect of age, $F(5,7)=42.8, p<.001$, a nonsignificant effect of hand, $(F<1)$ and a significant age by hand interaction, $F(5,7)=5.05, p=.028$. As Figure 2 shows, $\mathrm{LT}$ was longer for the right hand at 20 weeks, but longer for the left hand at 30 weeks. LT was calculated for each type of movement by hand at each age to explore the significance of this interaction.

\section{LT at 20 weeks}

At 20 weeks, where only approach and reaching movements are observed, LT of the right arm was longer than LT of the left hand. Figure $3 \mathrm{a}$ shows that 
(a)

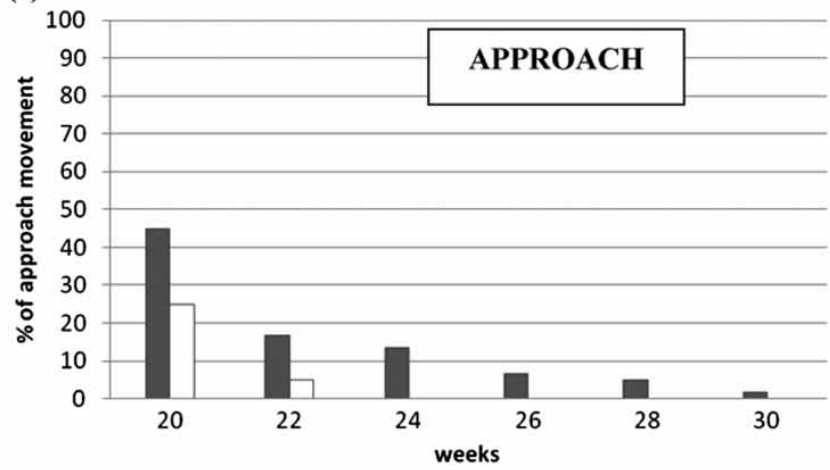

- LEFTHAND $\square$ RIGHT HAND

(b)

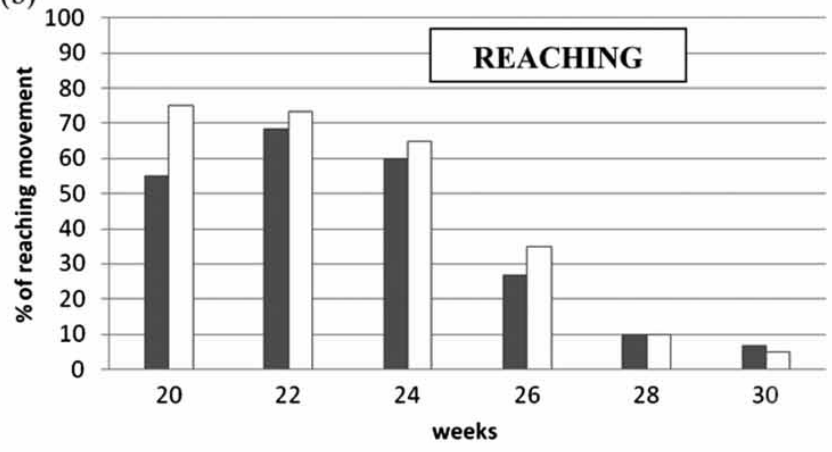

- LEFTHAND $\square$ RIGHTHAND

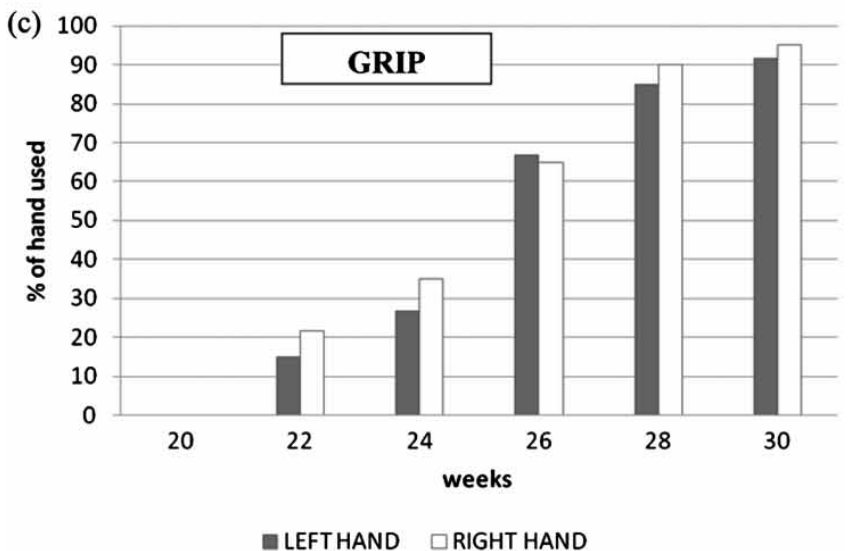

Figure 1. Age-related changes in approach (a), reaching (b) and grip (c) movements according to the hand used. 


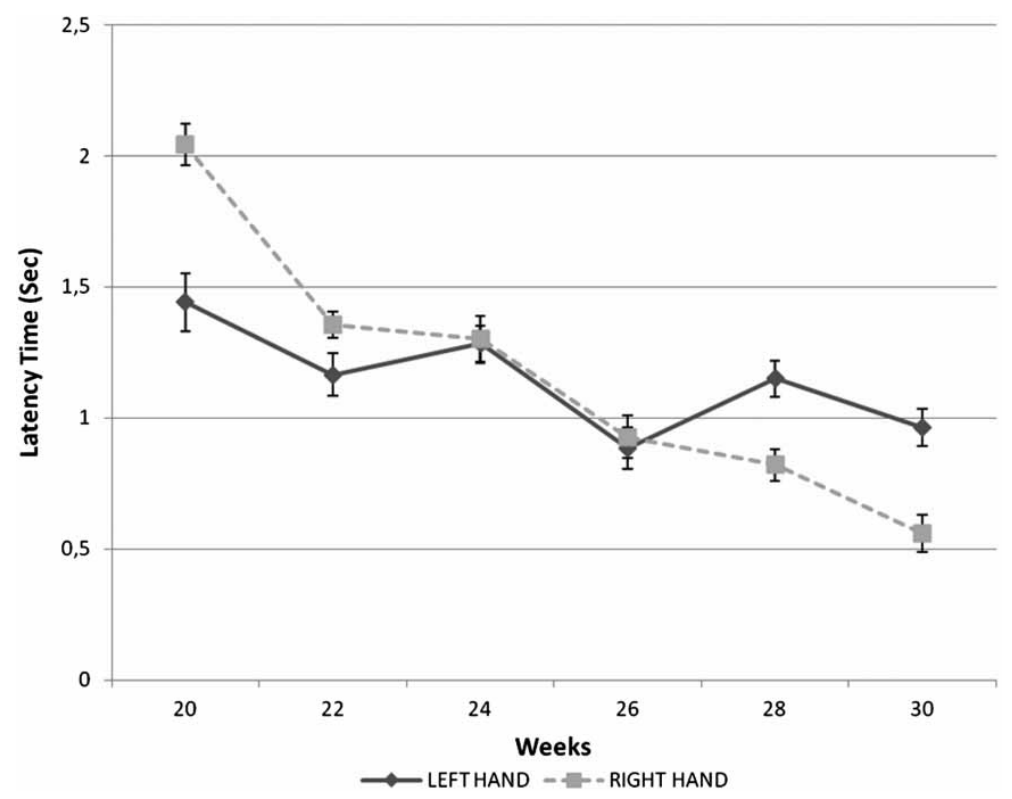

Figure 2. Latency time of left-handed and right-handed movements in babies from 20 to 30 weeks.

this difference in overall LT is mainly due to the approach movements. The repeated-measures analyses corresponding to Figure 3 show a hand effect: the right hand is slower than the left, $F(1,8)=31.5, p<.001$, a type of movement effect: overall approach slower than reaching, $F(1,8)=14.0$, $p=.006$, and a strong hand by type of movement interaction, $F(1,8)=65.3$, $p<.0001$. The hand effect was significant for approach movements, $t(8)=-7.8, p<.001$, but not for reaching movements, $t(11)=-0.90$, $p=.39$. Individual data of LT for approach movements show that in all 12 cases (Figure 3b) mean LT of the right arm was longer than mean LT of the left arm. For the left hand the reaching minus approach difference was positive and significantly different from $0, t(11)=4.41, p=.001$; for the right hand the reaching minus approach difference was negative and significantly different from $0, t(8)=6.54, p<.001$. This strongly suggests that approach movements with the right arm have a different meaning from approach movements of the left arm at 20 weeks of age.

\section{Reaching LT and grasping LT}

Analysis of LT for reaching movements at 20, 22, and 24 weeks only reveals an age effect, $F(2,10)=20.4, p<.001$, without significant right-left difference $(p=.61)$ or age by hand interaction $(p=.58)$ (Figure 4a). Detailed analyses show that reaching time decreased significantly from 20 to 22 
(a)

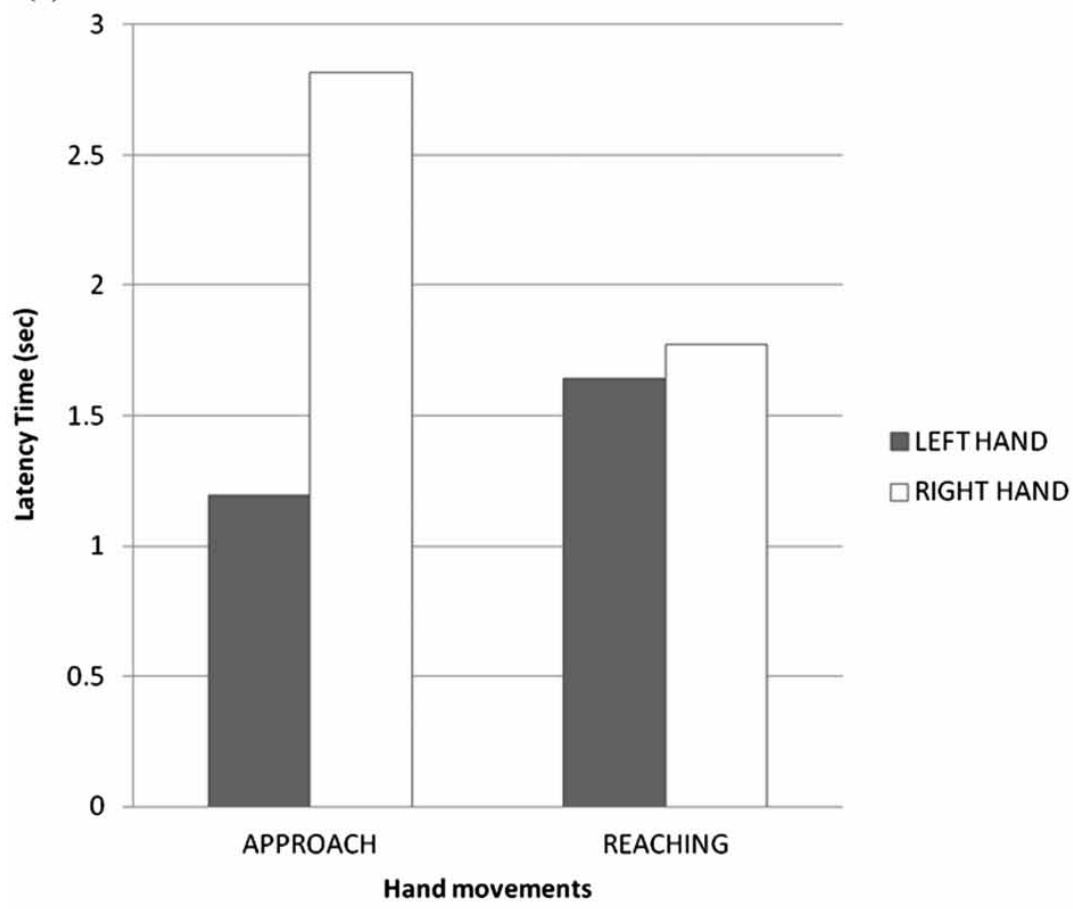

(b) $\quad(\mathrm{N}=12)$

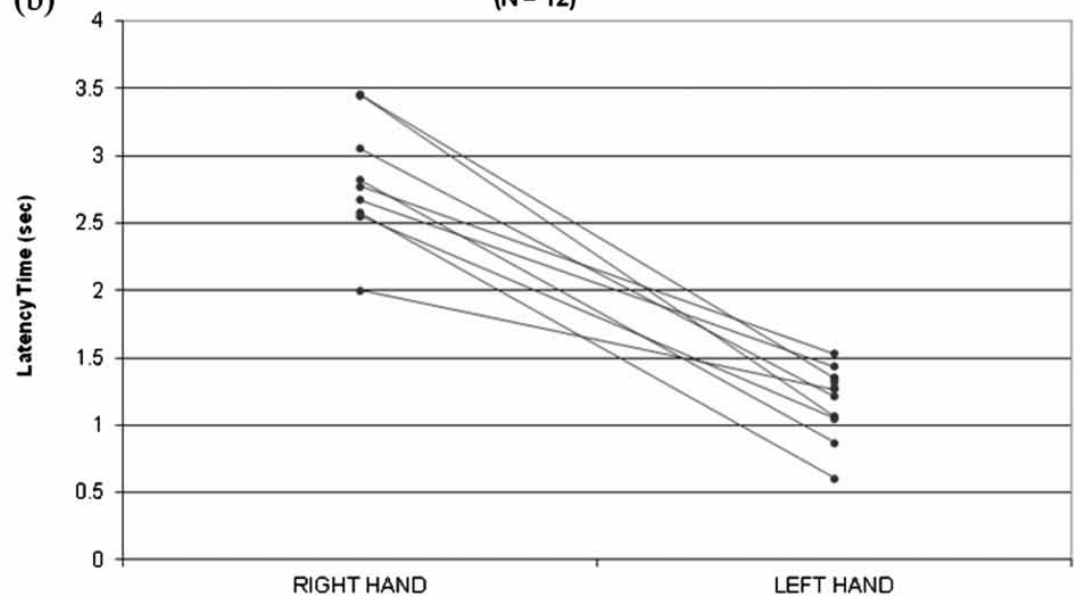

Figure 3. LT at 20 weeks: (a) comparison between approach and reaching movements and (b) individual data for approach movements by hand. 
weeks, $F(1,11)=26.9, p<.001$, and from 20 to 24 weeks, $F(1,11)=6.7$, $p=.03$, but not from 22 to 24 weeks, $F(1,11)=0.52, p=.49$.

Analysis of LT for grasping movements shows an age effect, $F(3,6)=7.2$, $p=.02$. Detailed analyses show that grasping time decreased significantly from 24 to 26 weeks, $F(1,8)=18.5, p=.003$, and from 28 to 30 weeks,

(a)

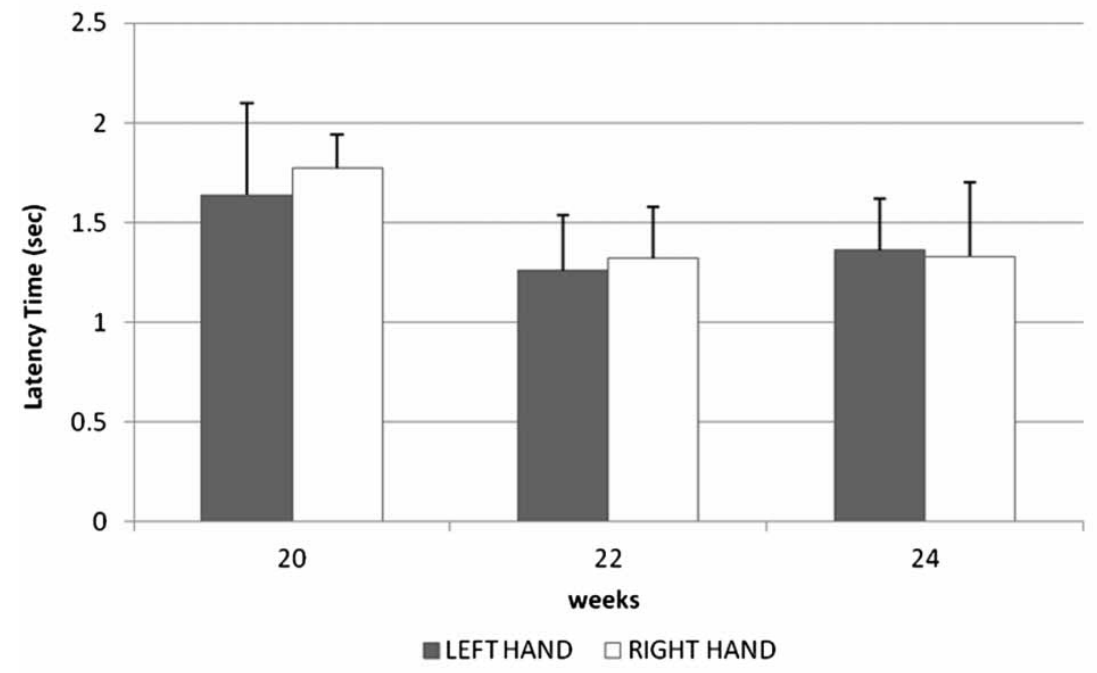

(b)

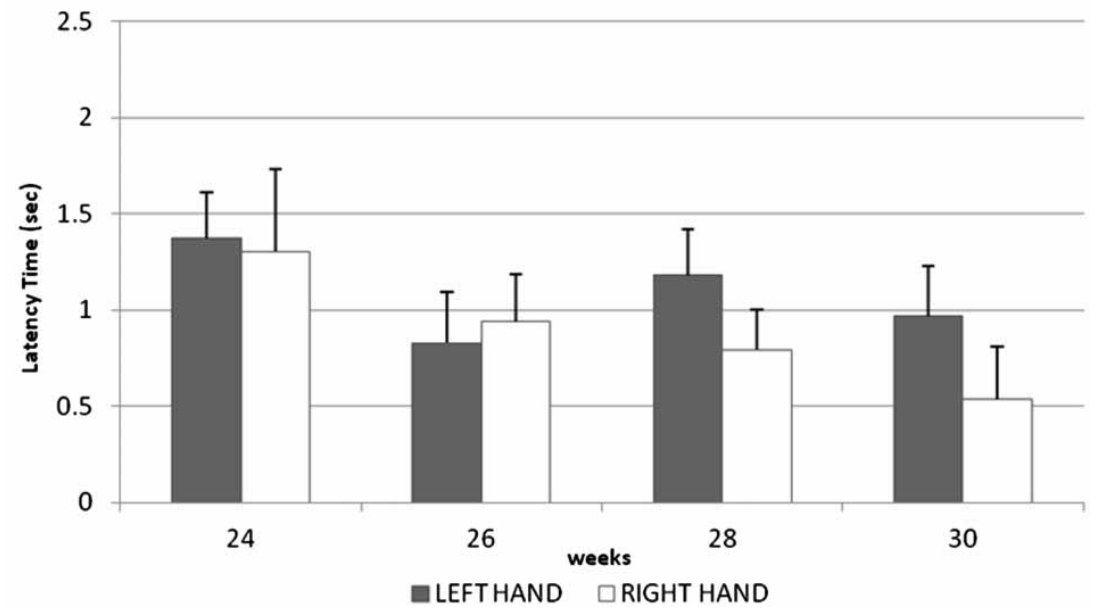

Figure 4. LT of (a) reaching movements from 20 to 24 weeks and (b) grasping movements from 24 to 30 weeks. 


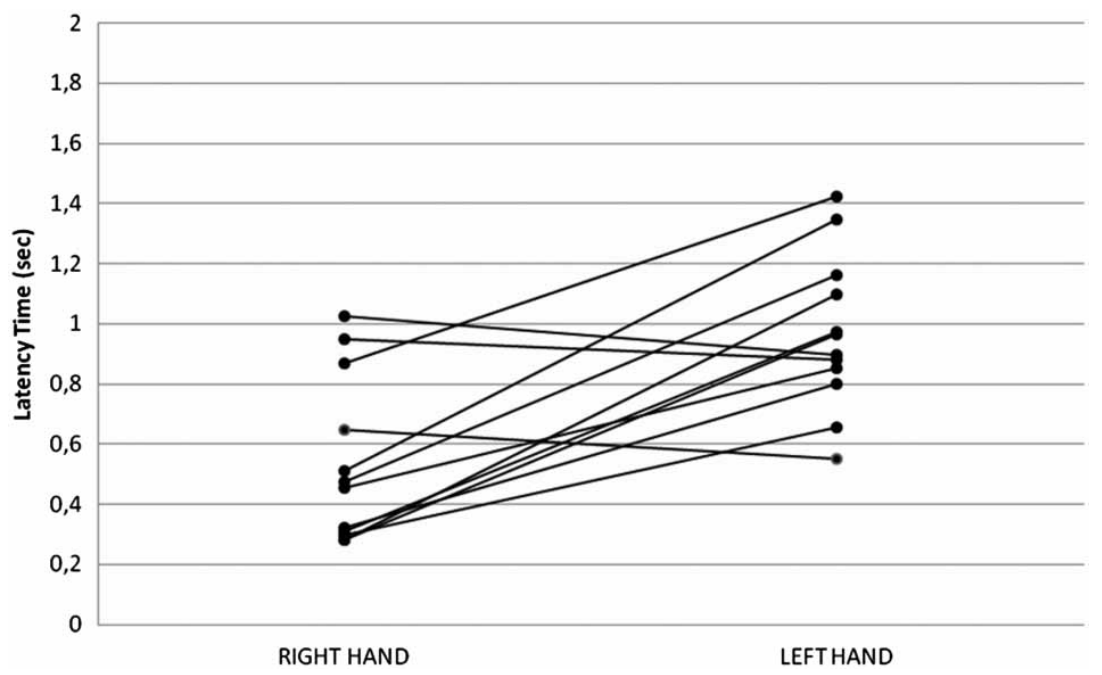

Figure 5. LT at 30 weeks: individual data for grasping movements by hand.

$F(1,11)=7.14, p=.02$, but not from 26 to 28 weeks, $F(1,10)=3.12, p=.11$. A hand effect was also revealed by the statistical analysis, $F(1,8)=12.15$, $p=.008$, but the age by hand interaction did not reach significance $(p=.10)$. The age effect was significant both for the left hand, $F(3,8)=5.7, p=.02$, and for the right hand, $F(3,7)=5.3, p=.03$. However, for the right hand a regular decrease in reaching time was observed, significant from 24 to 26 weeks, $t(9)=3.8, p=.004$, not significant from 26 to 28 weeks, $t(11)=1.5$, $p=.17$, and significant from 28 to 30 weeks, $t(11)=2.45, p=.03$. On the contrary, for the left hand the variation in reaching time was not linear, with important decrease from 24 to 26 weeks, $t(10)=4.6, p<.001$, significant increase between 26 and 28 weeks, $t(10)=-3.1, p=.01$, and not significant decrease between 28 and 30 weeks, $t(11)=1.75, p=.10$.

Figure 5 shows individual data for grasping LTs of each hand at 30 weeks. At 30 weeks the hand effect was significant, $t(11)=-4.32, p<.01-9 / 12$ babies showing shorter latencies for the right hand, the other 3 showing only mild difference between hands.

\section{DISCUSSION}

The present longitudinal design allowed us to specify the age of appearance of the three types of movements identified previously (Morange \& Bloch, 1996; Morange-Majoux \& Dellatolas, 2010) when a small object is placed laterally on a stand and the baby sits (the seated position is more effective in promoting reaching movements; Carvalho, Tudella, \& Savelsbergh, 2007). In 
accordance with previous investigations (Gesell \& Ames, 1947; Halverson, 1931; Hofsten, 1991; Mathew \& Cook, 1990; Michel, 2002), grip movements were completely absent at 20 weeks ( $41 / 2$ months), and represented more than $60 \%$ of all arm movements at 26 weeks, and more than $90 \%$ at 30 weeks, for both right and left hands. Approach movements of the right arm were observed mainly at 20 weeks (about $25 \%$ of all right arm movements), were rare at 22 weeks ( $5 \%$ of all right arm movements), and completely absent afterwards. Approach movements of the left arm represented $45 \%$ of all left arm movements at 20 weeks (the infant was doing less reaching with the left hand) and became rare but still present from 22 to 30 weeks. Reaching movements represented the majority of movements from 20 to 24 weeks, with no significant hand effect. In summary, when objects are placed laterally, a "laterality effect" of approach movements is observed between 20 and 30 weeks, more frequent with the left hand, confirming previous observations (e.g., Morange \& Bloch, 1996; Morange-Majoux \& Dellatolas, 2010).

This study also offers strong evidence that babies exhibit very early a latency phase of their movements, between $500 \mathrm{~ms}$ and 2 seconds, from the moment when they see the object to the moment when they start their hand movement. From 20 to 30 weeks latencies of right and left hand movements towards the object are different, according to age and to the type of movements. In our hypothesis these observed differences of LT reflect lateral differences in perceptual processing and hemispheric specialisation as in adults. Planning a movement takes into account the information of extrinsic properties, such as distance and direction, which define the spatial location of the objects in the environment, and intrinsic properties, such as size and shape, which define their identity (Arbib, 1981; Jeannerod, 1981; Loukopoulos, Engelbrecht, \& Berthier, 2001) and we can hypothesise that the longer the LT, the more perceptual information is gathered about the object. Thus, during the crucial period of 20-30 weeks where the onset of prehension undergoes profound modifications - going from approach to reaching and then grip in a coordinated hand-eye-object fashion-intrinsic properties will play a growing part during planning of the movement and LT will increase between 20 and 30 weeks. In other words, planning an approach movement (ballistic movement) could require less time for processing visual information than planning a reaching or grip movement. Nevertheless, we observed differently; LT decreased during this period, especially for the right arm. These results should be interpreted by taking into account the type of movement performed and the hand used:

(1) Concerning approach and reaching movements at 20 weeks, results showed that approach LT was shorter than reaching LT for the left hand, whereas the opposite was observed for the right hand. The high 
right hand LTs as early as 20 weeks indicate long planning, which leads us to consider right hand approach movements as rather unsuccessful reaching attempts.

(2) Concerning reaching and grip movements between 20 and 30 weeks, differences are observed in LTs for left and right grip. These results could be explained by the type of information that must be extracted; when the movement only needs extrinsic information, i.e., object localisation, the left hand has an advantage in performing an approach movement compared to the right hand, as shown by the short left hand LTs. When the movement becomes more complex, i.e., a need to have intrinsic information such as the size of the object, the LT increases to adapt its planning. However, if right hand approaches are pre-grip movements (unsuccessful reaching attempts) then right hand grip movements could require less time for programming than left hand movements, and the decrease in right hand LTs between 20 and 30 weeks could be caused by training and experience of the right hand, resulting in more rapid and efficient programming of the movement. In other words, right hand movements will become automatised before left hand movements. In conclusion, we suggest that babies exhibit a preparatory stage before the movement which allows them to extract critical and specific information about properties of the target object in order to specify the appropriate movement and the appropriate hand.

However, this period is marked by deep changes in motor control and motor experience, particularly the control of the trunk (Thelen \& Spencer, 1998; Van Der Fits, Klip, Eykern, \& Hadders-Algra, 1999). The emergence of novel sensory-motor experiences at these ages (progressive control of the arm, hand, fingers, trunk) could be associated with early developmental fluctuations in hand use and impact manual laterality (Corbetta, Thelen, \& Johnson, 2000). Thus these changes in LT could simply reflect changes in the ability to issue clean motor commands. If infants do intend to grasp the object with the right hand at 20 weeks, then longer LT would provide more time to gather proprioceptive information - about the hand, the head, the trunk, and fine-time motor commands to the extremities - in addition to gathering more information about the target. With age, part of the automatising may be reduction in the noise of the proprioceptive and motor systems. Some studies have shown that infants begin to plan their motor responses as a function of objects' physical properties at around 8 months old (adaptation of the shape of the hand to the shape of the object for example) and to adjust their grip configuration to the object size as early as 4 months (Newell, Scully, McDonald, \& Baillargeon, 1989; Von Hofsten \& Ronnqvist, 1988) and to limit their movements when they can't try to reach the object (Morange-Majoux \& Dellatolas, 2010). Even if the part of the 
proprioceptive information should not be underestimated, LT probably reflects an important part of exteroceptive information extraction.

These findings provide insight into the study of manual laterality by demonstrating that a manual specialisation (both in the perceptual and motor parameters) is present before reach and grip onset: the left arm/hand movement using extrinsic information to localise the object and right arm/ hand movement intrinsic information to prepare grasping and manipulation. More exactly, differences between the two hands for the type of movement and its planning will reflect hemispheric differences in the structural organisation of different cortical areas (Massion, Paillard, Schultz, \& Wiesendanger, 1983; Spinelli \& Jensen, 1982). Manual specialisation appears while children do not present clear manual preference. At 20-30 weeks infants' preferences are too variable across assessment conditions and age (Carlson \& Harris, 1985). Michel and Harkins (1986) found a stability of preference from 6 to 13 months but not before. As Michel (1998) explained, handedness is particularly difficult to assess in infants because it is manifested according to the level of the infant's manual ability. Instead, preferred hand use fluctuates widely between right and left over the weeks and months that follow the onset of voluntary reaching (Corbetta, Williams, \& Snapp-Childs, 2006). The left hand is more efficient at spatial adjustments (extrinsic information) requiring rapid proximal programming, and the right hand is more devoted to fine manual acts (intrinsic information) requiring long distal programming, as has been shown in adults (Jeannerod, 1986). This manual specialisation seems to be present as early as 4 months of age. These results show that manual specialisation appears before reach onset, contrary to studies suggesting that strong hand asymmetry emerges after reach onset (Baht \& Galloway, 2006; Corbetta \& Thelen, 1999; Lynch, Lee, Baht, \& Galloway, 2008; Michel, Sheu, \& Brumley, 2002; Michel et al., 2006; Rönnqvist \& Domellof, 2006). An old study by Caplan and Kinsbourne (1976) can be interpreted in this perspective; as early as 2 months of age, babies show an asymmetry of duration of grasp: they grip a rattle longer with the right hand than with the left hand. We propose that manual specialisation is the manifestation of hemispheric specialisation and emerges early in the development, whereas handedness is the manifestation of the lateralised body, where the subject must choose between two hands to apprehend objects; it thus emerges with the development of prehension. This choice is strongly influenced by the hemispheric specialisation - the right hand being the most efficient hand (left hemisphere) - to make a successful grip, which is the ultimate purpose in fine.

The current literature focuses more often on handedness than on manual specialisation in very young infants (Fagard, J., Spelke, \& von Hofsten, 2009; Ferre, Babik, \& Michel, 2010; Hinojosa, Sheu, \& Michel, 2003). This study shows that babies do not just manifest a fluctuating manual preference but 
also express a manual specialisation, reflecting hemispheric specialisation at an early stage of development.

Manuscript received 25 April 2011

Revised manuscript received 19 December 2011

First published online 31 May 2012

\section{REFERENCES}

Annett, M. (2004). Hand preference observed in large healthy samples: Classification, norms and interpretations of increased non-right-handedness by the right shift theory. British Journal Psychology, 95, 339-353.

Arbib, M. A. (1981). Perceptual structures and distributed motor control. In V. B. Brooks (Ed.) Handbook of physiology, vol. II (pp. 1449-1480). Bethesda, MD: American Physiological Society.

Bard, C., Hay, L., \& Fleury, M. (1990). Timing and accuracy of visually directed movements in children: Control of direction and amplitude components. Journal of Experimental Child Psychology, 50, 102-118.

Berthier, N. E. (1996). Learning to reach: A mathematical model. Developmental Psychology, 32, $811-823$.

Berthier, N. E., Clifton, R. K., McCall, D. D., \& Robin, D. J. (1999). Proximodistal structure of early reaching in human infants. Experimental Brain Research, 127, 259-269.

Bhat, A. N., \& Galloway, J. C. (2006). Toy-oriented changes during early arm movements: Hand kinematics. Infant Behavior and Development, 29, 358-372.

Boulinguez, P., Nougier, V., \& Velay, J.-L. (1991). Manual asymmetries in reaching movement control. I: Study of right-handers. Cortex, 37, 101-122.

Brown, J., Sepher, M. M., Ettlinger, G., \& Skreczek, W. (1986). The accuracy of aimed movements to visual targets during development: The role of visual information. Journal of Experimental Child Psychology, 41, 443-460.

Bruner, J. S. (1969). Eye, hand and mind. In D. Elkind \& J. Flavell (Eds.), Studies of cognitive development: Essays in honor of Jean Piaget (pp. 223-235). New York: Oxford University Press.

Caplan, P. J., \& Kinsbourne, M. (1976). Baby drops the rattle: Asymmetry of duration of grasp by infants. Child Development, 47, 532-534.

Carlson, D. F., \& Harris, L. J. (1985). Development of the infant's hand preference for visually directed reaching: Preliminary report of a longitudinal study. Infant Mental Health Journal, 6, $158-174$.

Carson, R. G. (1989). Manual asymmetries: Feedback processing, output variability and spatial complexity: Resolving some inconsistencies. Journal of Motor Behavior, 21, 38-47.

Carson, R. G., Chua, R., Elliott, D., \& Goodman, D. (1990). The contribution of vision to asymmetries in manual aiming. Neuropsychologia, 28, 1215-1220.

Carson, R. G., Chua, R., Goodman, D., Byblow, W. D., \& Elliott, D. (1995). The preparation of aiming movements. Brain and Cognition, 28, 133-154.

Carvalho, R. P., Tudella, E., \& Savelsbergh, G. J. P. (2007). Spatio-temporal parameters in infant's reaching movements are influenced by body orientation. Infant Behavior and Development, 30, $26-35$.

Corbetta, D., \& Thelen, E. (1999). Lateral biases and fluctuations in infants' spontaneous arm movements and reaching. Developmental Psychobiology, 34, 237-255.

Corbetta, D., Thelen, E., \& Johnson, K. (2000). Motor constraints on the development of perception-action matching in infant reaching. Infant Motor and Development, 23, 351-374. 


\section{8}

MORANGE-MAJOUX, LEMOINE, DELLATOLAS

Corbetta, D., Williams, J., \& Snapp-Childs, W. (2006). Plasticity in the development of handedness: Evidence for normal development and early asymmetric brain injury. Developmental Psychobiology, 48, 460-471.

DiFranco, D., Muir, D. W., \& Dodwell, P. C. (1978). Reaching in very young infants. Perception, 7 , 385-392.

Elliott, D., \& Chua, R. (1996). Manual asymmetries in goal-directed movement. In D. Elliott \& E. A. Roy (Eds.), Manual asymmetries in motor performance (pp. 143-158). Boca Raton, FL: CRC Press.

Elliott, D., Roy, E. A., Goodman, D., Carson, R. G., Chua, R., \& Maraj, B. K. V. (1993). Asymmetries in the preparation and control of manual aiming movements. Canadian Journal of Experimental Psychology, 47, 570-589.

Fagard, J. (1997). Motricité réactive versus motricité prédictive: Changements dans le geste d'atteinte de l'objet au cours de la première année [Reactive motricity vs predictive motricity: Reaching changes during the first year of life]. In J. Lorenceau, A. Streri, B. Victorri, \& Y. M. Vissetti (Eds.), Le mouvement: des boucles sensori-motrices aux représentations cognitives et langagières. Bonas: Actes de la sixième école d'été de l'Association pour la Recherche Cognitive.

Fagard, J., \& Lockman, J. J. (2005). The effect of task constraints on infants' (bi)manual strategy for grasping and exploring objects. Infant Behavior \& Development, 28, 305-315.

Fagard, J., Spelke, E., \& von Hofsten, C. (2009). Reaching and grasping a moving object in 6-, 8-, and 10-month-old infants: Laterality and performance. Infant Behavior \& Development, 32, $137-146$.

Fallang, B., Saugstad, O. D., Grogaard, J., \& Hadders-Algra, M. (2003). Kinematic quality of reaching movements in preterm infants. Pediatric Research, 53, 836-842.

Ferre, C. L., Babik, I., \& Michel, G. F. (2010). Development of infant prehension handedness: A longitudinal analysis during the 6- to 14-month age period. Infant Behavior \& Development, 33, 492-502.

Fish, J. D., \& Goodale, M. A. (1979). The effects of instructions to subjects on the programming of visually directed reaching movements. Journal of Motor Behavior, 21, 5-20.

Fisk, J. D., \& Goodale, M. A. (1985). The organization of eye and limb movements during unrestricted reaching to targets in contralateral and ipsilateral visual space. Experimental Brain Research, 60, 19-32.

Flowers, K. (1975). Handedness and controlled movement. British Journal of Psychology, 66, 39-52.

Gentilucci, M., Castiello, U., Corradini, M. L., Scarpa, M., Umiltà, C., \& Rizzolatti, G. (1991). Influence of different types of grasping on the transport component of prehension movements. Neuropsychologia, 29, 361-378.

Gesell, A., \& Ames, L. B. (1947). The development of handedness. Journal of Genetic Psychology, $70,155-175$.

Haaland, K. Y., \& Harrington, D. (1989). The role of the hemispheres in closed loop movements. Brain and Cognition, 9, 158-180.

Halverson, H. M. (1931). An experimental study of prehension in infants by means of systematic cinema records. Genetic Psychology Monographs, 10, 107-283.

Henry, F. M., \& Rogers, D. E. (1960). Increased response latency for complicated movements and a "memory drum" theory of neuromotor reaction. Research Quarterly of the American Association for Health, Physical Education and Recreation, 31, 448-458.

Hinojosa, T., Sheu, C.-F., \& Michel, G. F. (2003). Infant hand-use preferences for grasping objects contributes to the development of a hand-use preference for manipulating objects. Developmental Psychobiology, 43, 328-334.

Hodges, N. J., Lyons, J., Cockell, D., Reed, A., \& Elliott, D. (1997). Hand, space and attentional asymmetries in goal-directed manual aiming. Cortex, 33, 251-269. 
Jeannerod, M. (1981). Intersegmental coordination during reaching at natural visual objects. In J. Long \& A. Baddeley (Eds.), Attention and performance (pp. 153-168). Hillsdale, NJ: Lawrence Erlbaum Associates Inc.

Jeannerod, M. (1986). Mechanisms of visuomotor coordination: A study in normal and braindamaged subjects. Neuropsychologia, 24, 41-78.

Jeannerod, M., Paulignan, Y., \& Weiss, P. (1998). Grasping an object: One movement, several components. Novartis Foundation Symposium, 218, 5-16; discussion 16-20.

Johnson, M. H., Posner, M. I., \& Rothbart, M. K. (1994). Facilitation of saccades towards a covertly attended location in early infancy. Psychological Science, 5, 90-93.

Klatzky, R. L., Fikes, T. G., \& Pellegrino, J. W. (1995). Planning for hand shape and arm transport when reaching for objects. Acta Psychologica, 3, 209-232.

Loukopoulos, L. D., Engelbrecht, S. E., \& Berthier, N. E. (2001). Planning of reach-and-grasp movements: Effects of validity and type of object information. Journal of Motor Behavior, 33, 255-264.

Lynch, A., Lee, H. M., Baht, A., \& Galloway, J. C. (2008). No stable arm preference during the prereaching period: A comparison of right and left hand kinematics with and without a toy present. Developmental Psychobiology, 50, 390-398.

Massion, J., Paillard, J., Schultz, W., \& Wiesendanger, M. (Eds.). (1983). Neural coding of motor performance. New York: Academic Press.

Mathew, A., \& Cook, M. L. (1990). The control of reaching movements by young infants. Child Development, 61, 1238-1257.

McDonnell, P. M. (1975). The development of visually guided reaching. Perception and Psychophysics, 19, 181-185.

Michel, G. F. (1998). A lateral bias in the neuropsychological functioning of human infants. Developmental neuropsychology, 14, 445-469.

Michel, G. F. (2002). Development of infant handedness. In D. J. Lewkowicz \& R. Lickliter (Eds.), Conceptions of development (pp. 165-186). New York: Psychology Press.

Michel, G. F., \& Harkins, D. A. (1986). Postural and lateral asymmetries in the ontogeny of handedness during infancy. Developmental Psychobiology, 19, 247-258.

Michel, G. F., Sheu, C.-F., \& Brumley, M. R. (2002). Evidence of a right-shift factor affecting infant hand-use preferences from 7 to 11 months of age as revealed by latent class analysis. Developmental Psychobiology, 40, 1-13.

Michel, G. F., Tyler, A. N., Ferre, C., \& Sheu, C.-F. (2006). The manifestation of infant hand-use preferences when reaching for objects during the seven- to thirteen-month age period. Developmental Psychobiology, 48, 436-443.

Morange, F., \& Bloch, H. (1996). Lateralisation of the approach movement and the prehension movement in infants from 4 to 7 months. Early Development and Parenting, 5, 81-92.

Morange-Majoux, F., \& Dellatolas, G. (2010). Right-left approach and reaching arm movements of 4-month infants in free and constrained conditions. Brain and Cognition, 72, 419-422.

Morange-Majoux, F., Pezé, A., \& Bloch, H. (2000). Organisation of left and right hand movement in a prehension task: A longitudinal study from 20 to 32 weeks. Laterality, 5, 351-362.

Newell, K. M., Scully, D. M., McDonald, P. V., \& Baillargeon, R. (1989). Task constraints and infant grip configuration. Developmental Psychobiology, 22, 1-13.

Oldfield, R. C. (1971). The assessment and analysis of handedness: The Edinburgh Inventory. Neuropsychologia, 9, 97-114.

Pellegrino, J. W., Klatzky, R. L., \& McCloskey, B. P. (1989). Time course of preshaping for functional responses to objects. Journal of Motor Behavior, 21, 307-316.

Pratt, J., \& Abrams, R. A. (1996). Action-centred inhibition: Effects of distractors on movement planning and execution. Human Movement Science, 13, 245-254.

Rochat, P. (1992). Self-sitting and reaching in 5 to 8 month old infants: The impact of posture and its development on early eye-hand coordination. Journal of Motor Behavior, 24, 210-220. 
Rönnqvist, L., \& Domellof, E. (2006). Quantitative assessment of right and left reaching movements in infants: A longitudinal study from 6 to 36 months. Developmental Psychobiology, $48,444-459$.

Sidaway, B., Yook, D., \& Russell, D. (1999). Distribute control in rapid sequential aiming responses. Journal of Motor Behaviour, 31, 367-379.

Spinelli, D. N., \& Jensen, F. E. (1982). Plasticity, experience and resource allocation in motor cortex and hypothalamus. In C. D. Woody (Ed.), Conditioning (pp. 161-169). New York: Plenum.

Thelen, E., Corbetta, D., \& Spencer, J. (1996). Development of reaching during the first year: Role of movement speed. Journal of Experimental Psychology: Human Perception and Performance, 22, 1059-1076.

Thelen, E., \& Spencer, J. (1998). Postural control during reaching in young infants: A dynamic systems approach. Neuroscience Biobehavioral Reviews, 22, 507-514.

Todor, J. I., \& Doane, T. (1978). Handedness and hemispheric asymmetry in the control of the movement. Journal of Motor Behavior, 10, 295-300.

Van Der Fits, I. B. M., Klip, A. W. J., Eykern, L. A., \& Hadders-Algra, M. (1999). Postural adjustments during spontaneous and goal-directed arm movements in the first half year of life. Behavioral Brain Research, 106, 75-90.

Velay, J. L., Daffaure, V., Raphael, N., \& Benoit-Dubrocard, S. (2001). Hemispheric asymmetries and interhemispheric transfer in pointing depend on the spatial components of the movement. Cortex, 37, 75-90.

von Hofsten, C. (1982). Eye-hand coordination in the newborn. Developmental Psychology, 18, $450-461$.

von Hofsten, C. (1991). Structuring of early reaching movements: A longitudinal study. Journal of Motor Behavior, 23, 280-292.

von Hofsten, C., \& Rönnqvist, L. (1988). Preparation for grasping an object: A developmental study. Journal of Experimental Psychology: Human Perception and Performance, 14, 610-621. 